\title{
ConceptER -Uma ferramenta para criação e manutenção do Modelo Entidade-Relacionamento e geração automática de instruções SQL para banco de dados
}

\author{
Pedro V. Pequeno ${ }^{1}$, Emmerson S. Rita ${ }^{1}$, Jucimar B. Souza ${ }^{1}$, Marcelo C. Machado ${ }^{1}$ \\ ${ }^{1}$ Instituto Federal de Educação, Ciência e Tecnologia do Amazonas (IFAM) \\ viniciuspedro350@gmail.com, \\ \{emmerson.silva, jucimar.souza, marcelo.chamy\}eifam.edu.br
}

\begin{abstract}
The Entity-Relationship Model (ERM) is very important for the development of a database project. Systems that have a good planning, obeying the rules of the model, tend to respond to user requests more efficiently and quickly. Most Database Management Systems (DBMS) has a specific tool for creating the relational model of tables graphically. Thereby, the designer needs to adapt the ERM in the relational model. Observing this situation, the ConceptER tool is proposed here, which helps designers to construct, in drag and drop, the diagram and automatically maps it to SQL scripts, independent of the chosen DBMS.
\end{abstract}

Resumo. O Modelo Entidade-Relacionamento (MER) é muito importante para o desenvolvimento de um projeto de banco de dados. Sistemas que possuem um bom planejamento, obedecendo as regras do modelo, tendem responder as requisições dos usuários de maneira mais eficiente e com rapidez. A maioria dos Sistemas de Gerenciamento de Banco de Dados (SGBD) possui uma ferramenta específica para criação do modelo relacional de tabelas de forma gráfica. Desse modo o projetista precisa adaptar o MER no modelo relacional. Observando esta situação, propõe-se aqui a ferramenta ConceptER que auxilia os projetistas a construir, de maneira drag and drop, o diagrama e o mapear automaticamente para os scripts $S Q L$, independente do SGBD escolhido.

\section{Introdução}

Os dados tornaram-se importantes no dia a dia de empresas e das pessoas, uma vez que podem caracterizar um cliente e criar vantagens econômicas sobre outras empresas. Atualmente, existem grandes quantidades de informações que não podem ser armazenadas em papéis como antigamente. A tecnologia de banco de dados foi desenvolvida para suprir a dificuldade de guardar grandes volumes de informações e otimizar a recuperação destas.

O projeto de banco de dados é separado em três visões, cada uma com seu significado e importância. No desenvolvimento de um projeto desta modalidade utilizase o Modelo Entidade-Relacionamento (MER), que é um diagrama composto de figuras geométricas, em alguns casos rotuladas, que possuem um sentido para mapear a estrutura do banco de dados. 
O desenho, através das figuras, do MER é chamado de Diagrama de EntidadeRelacionamento (DER) e a partir dele é realizado o mapeamento para o modelo relacional, que consiste de representações de tabelas, e a partir dessas representações que são geradas as estruturas físicas, utilizando conjuntos de comandos na linguagem $S Q L$, chamados de scripts.

Um Sistema de Gerenciamento de Banco de Dados (SGBD) é um conjunto de programas que permite criar e manter vários bancos de dados[NAVATHE and ELMASRI 2011]. Os SGBDs, geralmente, possuem ferramentas para criação do modelo relacional de tabelas utilizando a configuração das estruturas graficamente geradas para os scripts $S Q L$, porém são proprietárias e não permitem exportar o projeto para outro SGBD.

Além disso, ferramentas que possibilitam o desenvolvimento do MER e a geração dos scripts são proprietárias e pagas. Observando esses problemas, propõe-se o ConceptER, uma ferramenta freeware que gera automaticamente os scripts $S Q L$ com base no MER.

\section{Objetivos}

O objetivo deste artigo é detalhar, explicar e demonstrar a importância e a utilização da ferramenta ConceptER no ensino e no desenvolvimento dos projetos utilizando banco de dados, assim como ela foi desenvolvida e possíveis trabalhos futuros.

\section{Trabalhos relacionados}

Alguns softwares apresentam funcionalidades semelhantes ao ConceptER que permitem a criação do MER, mas são limitados quando se trata de seguir a notação de [NAVATHE and ELMASRI 2011], que os professores de Cursos de Banco de Dados mais utilizam, na qual o ConceptER se baseia para a criação do MER.

O brModelo é uma ferramenta bastante utilizada freeware para modelagem de banco de dados proposta por [CÂNDIDO 2017], cujo objetivo é a criação de esquemas conceituais e lógicos, que permite a construção tanto do MER quando do MER estendido (MERE). O software disponibiliza a conversão do MER para o modelo relacional de tabelas e deste para os scripts $S Q L$. Porém, a ferramenta permite ao usuário alterar o modelo relacional de tabelas, assim não mantendo a integridade entre os diagramas.

\section{ConceptER}

O ConceptER é uma ferramenta gráfica e Open Source (link do fonte: https://github.com/ViniPequeno/ConceptER) que visa ajudar os projetistas de banco de dados, propondo um ambiente interativo para criação do DER usando recursos de arrastar e soltar. A ferramenta, ainda permite, exportar para imagem e para os scripts $S Q L$.

O mapeamento para os scripts é feito de forma automática. O usuário pode escolher entre salvar os scripts em um arquivo ou conectar direto com o SGBD desejado. A ferramenta procura a melhor forma de mapeamento usando uma série de condições para manter a integridade do MER para os scripts SQL. 
Mesmo a ferramenta não realizando o modelo relacional de tabelas, ela não recomenda ignorá-lo. O MR de tabelas representa os scripts SQL de forma gráfica numa melhor visualização até por ser independente do SGBD.

\section{Resultados e conclusões}

A ferramenta ${ }^{1}$ possui todos os requisitos funcionais concluídos, exportando o MER para os Scripts SQL, procurando manter as regras do modelo relacional descrito por Navathe.

A figura1 ilustra como pode ser criada uma entidade no ConceptER. Observe que no topo da tela existe uma paleta de ferramentas com os ícones que representam os recursos para desenvolver um MER. Assim o usuário clica sobre o retângulo, que representa uma entidade, e solta sobre a área de trabalho. Na sequência ele visualiza a tela propriedades à esquerda e preenche o nome da entidade.

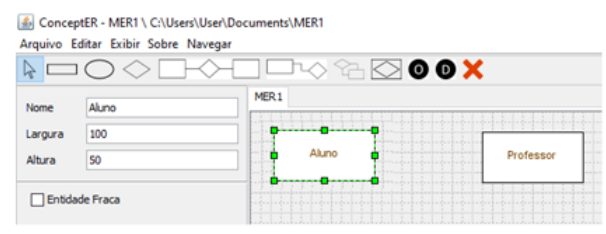

Figura 1. Exemplo de criação de entidades. Fonte própria.

$\mathrm{Na}$ figura 2 podem ser visualizados os atributos que foram adicionados as entidades. Observe que ao adicionar um atributo através do clique sobre a figura e arrastando para área de trabalho, a caixa de propriedade surge no lado esquerdo da tela onde o usuário pode definir o tipo de atributo: composto, multivalorado, atributo chave e outros.

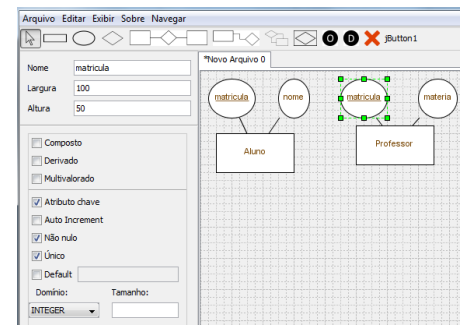

Figura 2. Exemplo de criação de atributos em suas entidades. Fonte própria.

A figura 3 apresenta a inserção de um relacionamento entre as entidades. O procedimento para inserir um relacionamento segue o mesmo princípio. Assim, no exemplo, obtemos uma relação onde um ou mais professores ensinam 1 ou mais alunos.

Quando o diagrama estiver finalizado, o usuário tem a opção de exportar para um SGBD. Neste caso o usuário seleciona qual o SGBD para exportar, conforme a figura 4. Informa a porta, usuário, nome do banco e após a confirmação, os scripts $S Q L$ serão executados e o banco será criado no SGBD especificado.

O ConceptER é uma ferramenta que tem a finalidade de criar o Modelo de Entidade Relacionamento dando suporte ao aluno que está aprendendo estes conceitos, e que

\footnotetext{
${ }^{1}$ Disponível na forma Open Source em https://github.com/ViniPequeno/ConceptER
} 


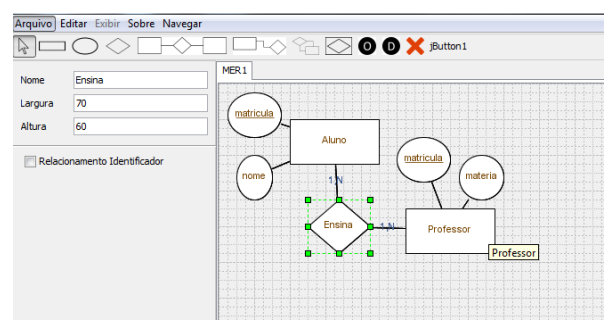

Figura 3. Exemplo de criação do relacionamento e suas ligações. Fonte própria.

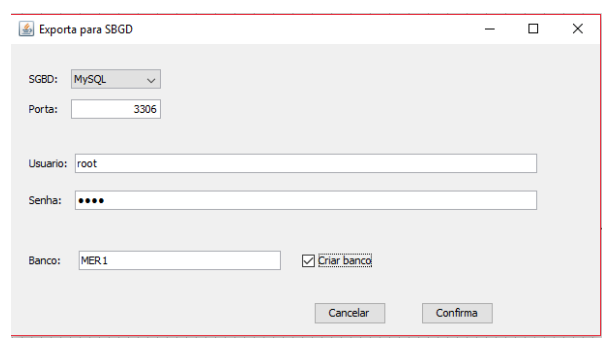

Figura 4. Tela de exportação para um SGBD específico. Fonte própria.

muitas das vezes, acaba recorrendo a recursos como editores de texto, editores gráficos, que não validam e não reforçam o aprendizado das aulas de banco de dados.

Como possíveis trabalhos futuros, pode-se apontar a possibilidade da criação do diagrama de tabelas, como ocorre nas ferramentas dos SGBDs e a montagem do DAO (Data Access Object) a partir do MER ou dos Scripts SQL para alguma linguagem Orientada a Objetos como Java, PHP e C++.

Após os testes feitos com as turmas do $2^{\circ}$ ano, percebeu-se que a ferramenta tem um grande potencial para auxiliar desenvolvedores no planejamento e construção dos seus banco de dados, principalmente por ser totalmente independente do sistema operacional e banco de dados trabalhado. Como é mostrado no gráfico abaixo, em teste com 40 alunos, tivemos $97,5 \%$ de aceitação do software, sendo utilizada como uma ferramenta CASE.

Figura 5. Gráfico de aceitação do software como ferramenta CASE

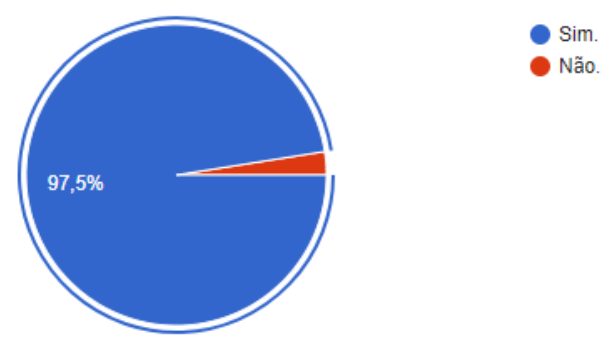

\section{Referências}

CÂNDIDO, C. H. (2017). brmodelo: Ferramenta de modelagem conceitual de banco de dados.

NAVATHE, S. B. and ELMASRI, R. (2011). Sistemas de banco de dados. Addison Wesley Longman, Inc. 